\title{
Nanotubos de carbono aplicados às neurociências: perspectivas e desafios
}

\section{Carbon nanotubes applied in neuroscience: prospects and challenges}

\author{
Virginia Oliveira ${ }^{1}$, Michele Munk Pereira1, Humberto de Mello Brandão², Marcos Antônio Fernandes Brandão¹, \\ Wagner Farid GattaZ ${ }^{3}$, Nádia Rezende Barbosa RaPoso ${ }^{1,3}$
}

${ }^{\top}$ Núcleo de Pesquisa e Inovação em Ciências da Saúde (NUPICS), Faculdade de Farmácia, Universidade Federal de Juiz de Fora (UFJF), MG.

2 Embrapa Gado de Leite, Juiz de Fora, MG.

3 Laboratório de Neurociências (LIM-27), Departamento e Instituto de Psiquiatria da Faculdade de Medicina da Universidade de São Paulo (FMUSP).

Recebido: 29/11/2010 - Aceito: 22/2/2011

\section{Resumo}

Introdução: Os nanotubos de carbono (NTCs) são os nanomateriais mais promissores para aplicação terapêutica em doenças neurodegenerativas. Aplicações potenciais incluem sistemas de liberação controlada de fármacos, interfaces elétricas e substratos para crescimento celular. Objetivo: Descrever o estado da arte e as perspectivas e desafios da aplicação dos NTCs nas neurociências. Métodos: Procedeu-se a uma busca sistemática nos indexadores Medline, Lilacs e SciELO, utilizando os descritores "carbon nanotubes", "drug delivery", "electrical interface", "tissue regeneration", "neuroscience", "biocompatibility" e "nanotechnology", devidamente agrupados. Resultados: A revisão da literatura evidenciou controvérsias nos estudos relativos à biocompatibilidade dos NTCs, embora tenha ratificado o seu potencial para a neuromedicina e neurociências. Conclusão: Os dados obtidos apontam a necessidade de estudos padronizados sobre as aplicações e interações dessas nanoestruturas com os sistemas biológicos.

Oliveira V, et al. / Rev Psiq Clín. 2011;38(5):201-6

Palavras-chave: Nanotubos de carbono, liberação controlada de fármacos, interface elétrica, regeneração tecidual, neurobiologia.

\begin{abstract}
Background: Carbon nanotubes (CNTs) are the most promising nanomaterials for therapeutic application in neurodegenerative diseases. Potential applications include systems for controlled drug delivery, electrical interfaces and substrates for cell growth. Objective: To describe the state of art, prospects and challenges of applying CNTs in neuroscience. Methods: There has been systematic search in Medline, Lilacs and SciELO, using the keywords "carbon nanotubes", "drug delivery", "electrical interface", "tissue regeneration", "neuroscience", "nanotechnology" and "biocompatibility", properly grouped. Results: The literature review showed controversies in studies on the biocompatibility of CNTs, although it has ratified its potential for neuromedicine and neuroscience. Discussion: These results highlight the need for modeling studies on the applications and interactions of nanostructures with biological systems.
\end{abstract}

Oliveira V, et al. / Rev Psiq Clín. 2011;38(5):201-6

Keywords: Carbon nanotubes, drug delivery, electrical interface, tissue regeneration, neurobiology.

\section{Introdução}

A nanotecnologia, segundo definição da ISO/TC 229, caracteriza-se pela aplicação do conhecimento científico para manipular e controlar a matéria na nanoescala, a fim de explorar as propriedades e fenômenos dependentes do seu tamanho e estrutura ${ }^{1}$. Indubitavelmente, essa é uma das áreas do conhecimento humano que mais se desenvolve ${ }^{2}$, beneficiando distintos segmentos, tais como alimentício, eletrônico, farmacêutico, biotecnológico, cosmético, médico-hospitalar e agrícola $^{3}$. A nanotecnologia promete avanços científicos e tecnológicos revolucionários na medicina, nas comunicações, na genética e na robótica ${ }^{4,5}$. Suas aplicações potenciais já descritas são amplas e diversificadas, e dentre elas se destacam como mais promissoras e de maior impacto a nanobiotecnologia, os nanofármacos e a nanoeletrônica ${ }^{6,7}$.

O grande diferencial dos materiais nanoestruturados é a potencialização de suas propriedades mecânicas, ópticas, magnéticas e químicas ${ }^{8}$. Isso ocorre, pois, na escala nanométrica, as estruturas revelam características peculiares e viabilizam produtos finais mais eficientes, leves, resistentes e, principalmente, de baixo custo 9 .

Dentre os vários nanomateriais, os nanotubos de carbono (NTCs) são os mais representativos, apresentando altíssimo potencial de inovação científica e tecnológica ${ }^{10}$. Esses materiais são excelentes candidatos para interface com sistemas neurais e têm despertado pesquisas para sua utilização na área das neurociências ${ }^{11}$. O interesse pelos NTCs nessa área reside na possibilidade de condutas terapêuticas revolu- cionárias para patologias como perdas auditivas neurossensoriais, doença de Parkinson, esclerose múltipla e doença de Alzheimerin,13. Vários estudos vêm sendo conduzidos com esse intuito, como a utilização de NTCs como substrato para regeneração neural ${ }^{14,15}$, próteses neurais capazes de controlar distúrbios motores ${ }^{16,17}$ ou traduzir sinais de dispositivos externos em ações neuronais específicas ${ }^{18,19}$, além de sistemas de liberação controlada ou direcionador de fármacos e biomoléculas para facilitar a transposição da barreira hematencefálica ${ }^{20}$.

\section{Objetivo}

O objetivo desta revisão é descrever o estado da arte e as perspectivas e desafios da aplicação dos NTCs nas neurociências.

\section{Métodos}

Procedeu-se a uma revisão atualizada da literatura científica indexada nas bases de dados Medline, Lilacs e SciELO, até o período de novembro de 2010. Os descritores utilizados para o levantamento bibliográfico foram: "carbon nanotubes", "drug delivery", "electrical interface", "tissue regeneration", "neuroscience", "biocompatibility" e "nanotechnology", devidamente agrupados. Foram realizadas análises concisas dos artigos, abordando seus aspectos relevantes para elaboração do presente estudo. 


\section{Resultados}

\section{Considerações gerais}

Os artigos encontrados destacam as características únicas dos nanomateriais, as quais possibilitam sua interação específica com sistemas biológicos e viabilizam sua utilização no diagnóstico e tratamento de doenças neurodegenerativas. Abordam, de forma geral, a utilização dos NTCs na liberação controlada de fármacos, engenharia de tecidos e interface elétrica com neurônios. Outros estudos destacam a compatibilidade dessas nanoestruturas com sistemas biológicos, ressaltando sua possível toxicidade. Foi observado que pesquisas envolvendo a aplicação da nanotecnologia nas neurociências ainda estão em fases iniciais, todavia os resultados preliminares são encorajadores.

\section{Nanotubos de carbono}

Os materiais grafíticos têm sido amplamente estudados desde o século passado, em virtude de suas propriedades e aplicabilidade industrial. Os NTCs foram sintetizados pela primeira vez em 1991 por Sumio Iijima, e sua descoberta representou grande evolução científica, sugerindo aplicações tecnológicas e especulações teóricas excepcionais, devidas, principalmente, ao seu comportamento eletrônico singular ${ }^{21}$.

Os NTCs são uma forma alotrópica do carbono caracterizada pelo enrolamento de uma ou várias folhas de grafeno de forma concêntrica e cilíndrica e com cavidade interna oca ${ }^{22}$. Podem ser classificados, quanto ao número de camadas, em NTCs de camada única (SWCNTs - "single-walled carbon nanotubes") ou de camadas múltiplas (MWCNTs - "multi-walled carbon nanotubes")23. O tamanho, geralmente, varia de 0,4-2 nm de diâmetro para os SWCNTs e de 2-100 nm para MWCNTs, enquanto seu comprimento pode ser de $1-100 \mu \mathrm{m}^{11}$.

Os NTCs são materiais estratégicos com grande interesse tecnológico, por causa principalmente de sua estrutura singular, a qual lhe confere um conjunto peculiar de propriedades mecânicas, ópticas, térmicas, químicas e eletrônicas ${ }^{24}$. A versatilidade desses materiais permite que sejam explorados em diferentes áreas de pesquisa e aplicação ${ }^{25}$.

$\mathrm{Na}$ nanomedicina, a busca pelo diagnóstico mais preciso, a regeneração de tecidos e a administração controlada de fármacos configuram-se como objetivos principais nas pesquisas e aplicações dos $\mathrm{NTCs}^{9,26,27}$. Nessa área, eles atuam como carreadores de fárma$\cos ^{28-30}$, próteses neurais ${ }^{16,18}$, marcadores biológicos e vetores de DNA na terapia gênica ${ }^{31,32}$. Os NTCs têm sido, ainda, amplamente estudados para a terapêutica de neoplasias e doenças neurodegenerativas ${ }^{15}$.

\section{Funcionalização de nanotubos de carbono}

Os NTCs podem ser manipulados por meio da funcionalização, ou seja, adsorção ou ligação de átomos ou moléculas às suas paredes ou pontas. A funcionalização torna os NTCs mais biocompatíveis e, portanto, facilita a sua interação com moléculas orgânicas, biológicas ou com outros grupos químicos, como fármacos ou DNA $\mathrm{DN}^{33,34}$.

A funcionalização com a adsorção de grupos químicos como a hidroxila torna os nanotubos solúveis em meio aquoso e facilita a interação com macromoléculas celulares ${ }^{35}$. Além disso, a funcionalização de NTCs resulta em menor toxicidade a células em culturas ${ }^{36}$, aumentando, assim, o seu potencial de aplicação nas áreas biológicas e médicas ${ }^{37}$.

\section{Biocompatibilidade dos nanotubos de carbono}

Embora a aplicabilidade desses materiais esteja em franco processo de exploração, estudos relativos à sua biocompatibilidade ainda são escassos e, por vezes, controversos ${ }^{38}$. Pouco se conhece acerca dos efeitos dos NTCs sobre a saúde humana e o meio ambiente, apesar da sua crescente disponibilidade comercial25.
Estudos científicos demonstraram que altas concentrações de nanopartículas podem gerar espécies reativas ao oxigênio após serem introduzidas nas células ${ }^{39,40}$, as quais danificam bases nitrogenadas e levam à mutação do DNA, induzindo a apoptose. Além disso, foram descritos comprometimento da viabilidade celular e função mitocondrial, aumento dos níveis de $\mathrm{LDH}$ por lesão da membrana celular, diminuição da glutationa reduzida, aumento de interleucina-8 e dos níveis de peroxidação lipídica ${ }^{41}$.

Belyanskaya et al.42 avaliaram os efeitos dos SWCNTs em células de adenocarcinoma de pulmão (A549) e verificaram, por meio do ensaio de MTT, significativas perdas de viabilidade celular. De Nicola et al.43 também observaram efeitos tóxicos resultantes da incubação de MWCNTs com monócitos, tais como: redução da viabilidade celular e aumento do índice apoptótico, cujas extensões se mostraram dependentes da concentração de MWCNTs e do tempo de exposição.

Em queratinócitos humanos, os SWCNTs provocaram aumento do estresse oxidativo e inibição da proliferação celular ${ }^{39}$, além de indução de apoptose, redução da adesão celular e aumento da resposta inflamatória. Estudos in vivo, nos quais ratos inalaram NTCs, evidenciaram injúria pulmonar, com formação de granulomas multifocais ${ }^{44}$. Adicionalmente, Shvedova et al.$^{45}$ demonstraram que as SWCNT se acumulam no pulmão, promovendo rápida formação de tecido granulomatoso e fibrose local. Segundo Li et al. ${ }^{46}$, a exposição respiratória a elevadas concentrações de SWCNTs provoca não só toxicidade pulmonar, como também efeitos vasculares relacionados a alterações mitocondriais oxidativas, além de formação acelerada de ateromas.

Shvedova et al. ${ }^{47}$ relataram alterações estruturais e morfológicas em cultura de queratinóticos humanos expostos a SWCNTs, além de formação de espécies reativas, acúmulo de produtos de peroxidação lipídica e redução da viabilidade, indicando toxicidade dérmica desses materiais. Entretanto, Yehia et al.48 demonstraram que SWCNTs não apresentam efeitos tóxicos in vitro para células epiteliais humanas, confirmando resultados anteriores, nos quais não foram observados riscos alérgicos e irritação cutânea a partir de exposição dérmica a essas estruturas ${ }^{49}$.

A funcionalização mostra-se determinante para a biocompatibilidade dessas estruturas ${ }^{34}$. Zhang et al. ${ }^{50}$ relataram que SWCNTs funcionalizados mantêm a viabilidade celular em cultura de queratinócitos humanos, enquanto outros grupos demonstraram que a funcionalização facilita a solubilização e a intracelularização dos NTCs ${ }^{51,52}$. Kagan et al. ${ }^{53}$ observaram, ainda, que alguns tipos de funcionalização podem potencializar o reconhecimento, favorecendo sua eliminação.

O grau de toxicidade dos NTCs depende do tipo de célula analisado, podendo ser influenciado pelo teste de toxicidade empregado, pela estrutura, estado de agregação e grau de pureza dos NTCs e, principalmente, pela funcionalização aplicada, o que explica a controvérsia dos estudos descritos e impede a comparação dos resultados encontrados $39,54,55$. A toxicidade dos NTCs pode estar relacionada com a presença de resíduos do metal de transição utilizado como catalisador no processo de síntese, principalmente ferro e níquel. Esses metais são promotores efetivos do estresse oxidativo das células, tecidos e biofluidos ${ }^{56}$. Fenoglio et al. ${ }^{57}$ sugeriram que o estresse oxidativo gerado pelos NTCs em culturas celulares está associado às impurezas de catalisadores metálicos em sua superfície. Pulskamp et al..$^{56}$ ratificaram essa teoria ao demonstrarem ausência de espécies reativas em culturas com NTCs purificados.

Como pode ser observado nos estudos supracitados, a maioria dos ensaios envolvendo a toxicidade dos NTCs tem se concentrado em células e tecidos mais suscetíveis ao contato imediato com essas partículas, como o sistema respiratório e o tecido epitelial ${ }^{53}$. Dados referentes à neurotoxicidade dos NTCs são ainda mais escassos, no entanto eles vêm sendo potencialmente estudados como substrato para regeneração neural ${ }^{14,15}$, bem como para próteses neurais ${ }^{16,17} \mathrm{e}$ sistemas de carreamento de fármacos ${ }^{20,58}$.

Experimentos recentes mostraram que partículas nanométricas podem ser conduzidas até o cérebro por meio dos neurônios olfativos ou da circulação sanguínea, fazendo com que a neurotoxicidade des- 
ses materiais seja uma questão importante ${ }^{59,60}$. Os nervos sensoriais da pele também constituem uma porta de entrada potencial para os NTCs alcançarem o SNC, segundo relataram Oberdörster et al. ${ }^{61}$.

Estudos específicos conduzidos com NTCs em cultura de células nervosas demonstraram que eles exercem influência negativa sobre a cultura, induzindo efeitos tóxicos agudos, redução da proliferação celular e alteração da condutividade iônica e do potencial de repouso da membrana ${ }^{25}$.

A compatibilidade com células eletricamente ativas é a chave para o sucesso dos NTCs na neurotecnologia. Entretanto, experimentos utilizando tais nanopartículas como suporte para proliferação neuronal revelaram crescimentos celulares alterados ${ }^{62,63}$. Além disso, estudos sugerem que os NTCs alteram as funções neuronais cálcio-dependentes, mediante a obstrução física dos canais iônicos ${ }^{64}$.

Por outro lado, Mazzatenta et al. ${ }^{65}$ evidenciaram em cultura de neurônios hipocampais que SWCNTs são capazes de estimular a atividade do circuito cerebral, sem observar quaisquer alterações na proliferação, morfologia ou adesão celular, decorrentes do contato com os nanomateriais. Lovat et al. ${ }^{17}$ também relataram a eficácia dos NTCs como superfícies de crescimento para neurônios, bem como sua influência no aumento da transmissão do sinal neuronal.

A avaliação desses resultados, por vezes antagônicos, evidencia a necessidade de padronização dos protocolos de toxicidade e a determinação de parâmetros a serem utilizados nos ensaios, como concentração e tempo de exposição celular.

\section{Doenças neurodegenerativas}

Doenças neurodegenerativas (DNs) são aquelas que acometem o SNC, sendo caracterizadas pela morte neuronal persistente, com evolução gradual e progressiva. Em geral, manifestam-se nos idosos, causando distúrbios cognitivos, dificuldades motoras e déficit de resposta sensorial ${ }^{66}$. Dentre as DNs, a doença de Alzheimer (DA) e a doença de Parkinson (DP) são as mais incidentes e para as quais ainda não existe tratamento efetivo ${ }^{67}$.

O diagnóstico e o tratamento das DNs representam um desafio para a neuromedicina. A complexidade do SNC e a restrição de acesso pela barreira hematencefálica são fatores que contribuem para esse quadro ${ }^{68}$. Atualmente, as abordagens terapêuticas limitam-se ao alívio sintomático, sendo incapazes de impedir ou compensar a perda neuronal e a progressão da doença ${ }^{69,70}$. Quanto ao diagnóstico, o parecer definitivo só é obtido por autópsia após a morte do paciente ${ }^{71}$. $\mathrm{O}$ advento da nanotecnologia trouxe novas perspectivas tanto para o diagnóstico quanto para a neuroterapia dessas DNs. Vislumbra-se o desenvolvimento de kits diagnósticos, técnicas de regeneração tecidual e vetores de fármacos, DNA, RNA e proteínas para a terapia gênica, além de dispositivos capazes de interceptar e alterar a transmissão de sinais neuronais, o que abre novas perspectivas para as condutas clínicas no campo das neurociências ${ }^{31,72}$.

\section{Aplicações potenciais de NTCs no tratamento de DNs}

\section{Sistemas de liberação controlada de fármacos}

Devido às limitações impostas pela barreira hematencefálica, sistemas convencionais de carreamento de fármacos não são eficientes no tratamento de DNs. Abordagens alternativas como administração transcraniana e transnasal ou agentes perturbadores da permeabilidade da membrana hematencefálica têm efeitos adversos e não refletem melhorias no quadro clínico das $\mathrm{DNs}^{73}$. A estratégia da nanotecnologia para conduzir biomoléculas e fármacos ao SNC consiste em utilizar sistemas de carreamento nanométricos que interajam seletivamente com receptores de transporte da barreira hematencefálica e libere seu conteúdo no alvo desejado mediante algum estímulo elétrico, magnético, químico ou térmico ${ }^{74,75}$, induzindo respostas fisiológicas diretas e minimizando os efeitos colaterais ${ }^{66}$. Além de suas dimensões nanométricas, que viabilizam a internalização no SNC, os nanocarreadores protegem os fármacos e moléculas bioativas da degradação metabólica e do sistema imunológico ${ }^{76}$.
Vários sistemas nanoestruturados de carreamento de bioativos até o SNC já estão em estágios avançados de pesquisa, com resultados farmacocinéticos promissores, a saber: nanopartículas poliméricas ${ }^{77}$, nanoesferas poliméricas ${ }^{78}$, nanomicelas poliméricas ${ }^{79}$ e lipossomas ${ }^{80}$.

Pesquisas com NTCs ainda estão em fases preliminares, mas já demonstram o seu potencial para atuar como nanocarreadores de genes, fármacos ou biomoléculas ${ }^{81}$. Shi Kam et al..$^{82}$ mostraram que nanotubos funcionalizados são capazes de atravessar as membranas celulares por endocitose ou difusão passiva. Adicionalmente, Tosi et al. ${ }^{8}$ obtiveram resultados satisfatórios de permeabilização dos NTCs na barreira hematencefálica de ratos por meio de funcionalização com ácido siálico. Vergoni et al. ${ }^{20}$ também demonstraram que a biodistribuição de nanopartículas conjugadas a biomoléculas, no SNC de ratos, foi de $15 \%$ da dose administrada, aproximadamente duas vezes maior que o alcançado por outros carreadores.

Dessa forma, as nanoestruturas mostram-se promissoras para o carreamento de fármacos e neurotransmissores específicos para o tratamento das DNs, tais como a DP, na qual os níveis de dopamina podem ser restabelecidos no cérebro pela liberação controlada desse neurotransmissor.

\section{NTCs como substratos para o crescimento celular}

A regeneração do tecido neuronal tem recebido especial atenção científica na área das neurociências, sendo fator determinante para o avanço terapêutico das DNs. Uma estratégia promissora é a implantação de suportes nanométricos promotores do crescimento axonal e do desenvolvimento de neuritos. No entanto, o sucesso das técnicas de reparo neural depende de um suporte sintético biocompatível análogo à matriz extracelular biológica, imunologicamente inerte, condutor e capaz de garantir as condições químicas, topográficas e elétricas necessárias à adesão e à proliferação das células nervosas ${ }^{83}$.

O tamanho, a forma, a flexibilidade e a condutividade elétrica dos NTCs são características favoráveis ao seu emprego como suporte de crescimento neuronal ${ }^{11}$. Em 2000, Mattson et al. ${ }^{63}$ relataram, pela primeira vez, a possibilidade de utilização dos NTCs como suporte para crescimento de neurônios. Eles demonstraram que neurônios hipocampais semeados em NTCs sobreviveram e continuaram crescendo por mais de oito dias em cultura, exibindo neuritos múltiplas, com extensas ramificações. Hu et al. ${ }^{62}$ ratificaram esses achados, identificando cones de crescimento, neuritos e ramificações em neurônios hipocampais de rato cultivados em MWCNTs. Estudos subsequentes mostraram que aspectos como crescimento, adesão e diferenciação dos neurônios cultivados em NTCs foram satisfató$\operatorname{rios}^{84,85}$. O sucesso do emprego dessas nanopartículas como suporte de crescimento celular deve-se, principalmente, à sua grande área de superfície, o que aumenta a adsorção de proteínas adesivas, tais como fibronectina e vitronectina, responsáveis pela mediação das interações células-substrato ${ }^{83}$.

Segundo Matsumoto et al. ${ }^{86}$, o crescimento dos neurônios em cultura pode ser estimulado com o uso de nanotubos funcionalizados com fatores de crescimento, tais como fator de crescimento neuronal (NGF) e fator neurotrófico derivado do cérebro (BDNF). A funcionalização com outras moléculas biologicamente ativas, como a caderina e a laminina, que aumentam a adesão das células ao substrato, também demonstrou favorecer o crescimento neuronal ${ }^{87}$. A manipulação da carga superficial dos nanotubos por meio de modificações covalentes também interfere nas características da cultura. Hu et al..$^{62}$ testaram o crescimento de neurônios hipocampais sobre NTCs funcionalizados com diferentes grupos químicos que lhes conferiam cargas superficiais positivas, neutras ou negativas e observaram maior quantidade de cones de crescimento, bem como neuritos com ramificações mais extensas nas culturas sobre substratos com carga positiva. Essas observações sugerem que os NTCs podem ser quimicamente modificados para a modulação do crescimento neuronal.

A viabilidade e a manutenção das características morfológicas das células em cultura são fatores fundamentais para que um material 
seja utilizado como substrato de cultivo. No entanto, no caso dos neurônios, é necessário mais que isso. $\mathrm{O}$ material deve garantir que sinapses funcionais se estabeleçam e não pode interferir negativamente nas propriedades eletrofisiológicas neuronais.

\section{Interface elétrica NTCs-neurônios}

Dispositivos de interface neural são eletrodos em íntimo contato com o sistema neurobiológico, capazes de restaurar a função neuronal perdida em virtude de uma patologia. A regeneração tecidual mediada por essas interfaces consiste basicamente em fornecer suporte físico e estímulos moleculares para orientar a cicatrização do tecido ${ }^{88}$. A concepção de interfaces neurais é um dos avanços mais significativos da nanoneurociência, que possibilitou o desenvolvimento de dispositivos implantáveis no SNC para controlar distúrbios motores ${ }^{16}$, resistência a medicamentos psiquiátrico ${ }^{89}$ e tradução de processos cerebrais voluntários em ações específicas por meio de dispositivos externos $^{18,19}$. Contudo, avanços clínicos nessa área ainda são limitados por problemas recorrentes, tais como baixa aderência neuronal e instabilidade de sinal elétrico, fazendo-se necessário encontrar materiais que eficientemente interajam com o tecido neural sem lhe causar danos ${ }^{90}$.

Os eletrodos atualmente utilizados para implantes cerebrais são metálicos e têm impedância relativamente alta, além de baixa capacidade de transferência de carga. As propriedades elétricas e mecânicas dos NTCs podem melhorar o desempenho desses implantes, possibilitando a sua utilização como interface com neurônios para restabelecer a atividade neuronal ${ }^{13}$. No entanto, a exploração dos NTCs em tais dispositivos biomédicos requer conhecimento acerca de suas ações sobre os neurônios, principalmente no que tange à excitabilidade neuronal, alterações na condutância iônica e em vias de sinalização intracelular, a fim de minimizar interações indesejáveis ${ }^{65}$.

A biocompatibilidade neuronal dos NTCs já foi demonstrada em vários estudos, e a aderência, a viabilidade e o crescimento dos neurônios podem ser modulados pela funcionalização das nanopartículas. Lovat et al. ${ }^{17}$ foram os primeiros a abordar os efeitos de substratos de NTCs sobre o comportamento elétrico dos neurônios em cultura. Eles observaram, após oito dias em cultivo, aumento de seis vezes da atividade elétrica neuronal sobre MWNTCs em comparação com o grupo controle - superfícies lisas de vidro. Efeitos similares foram encontrados por Mazzatenta et al. ${ }^{65}$ usando SWCNTs, que relataram aumento da frequência de correntes pós-sinápticas espontâneas em neurônios hipocampais, além de maior excitabilidade. Dessa forma, os NTCs são capazes de despolarizar e hiperpolarizar a membrana neuronal por meio de interações sinápticas, permitindo estimulação contínua e criação de novos circuitos neuronais. Ainda neste estudo, imagens de microscopia eletrônica de varredura mostraram neurônios morfologicamente normais e aderidos ao substrato, revelando íntimo contato neurônio-nanotubo e estabilidade da interface elétrica.

Cellot et al. ${ }^{11}$ verificaram o crescimento de neurônios hipocampais sobre um substrato com alta condutividade elétrica e outro com estrutura nanométrica, a fim de esclarecer a influência dessas duas propriedades físicas presentes nos NTCs sobre a excitação neuronal. Em nenhum dos dois suportes foram encontrados padrões de crescimento semelhantes aos obtidos com NTCs, mostrando que os efeitos desse substrato são específicos e não são facilmente reprodutíveis. Eles ainda investigaram a natureza das interações neurônio-NTC e propuseram que NTCs estimulam a transmissão do sinal neuronal por meio da formação de vias adicionais de acoplamento elétrico entre os componentes neuronais somáticos e dendríticos. Análises das propriedades eletrofisiológicas das membranas de neurônios hipocampais cultivados em NTCs não revelaram qualquer alteração de condutividade iônica ${ }^{17,65}$, embora estudos mais recentes tenham demonstrado alterações das funções celulares cálcio-dependentes, sugerindo que NTCs podem ocluir fisicamente os canais iônicos ${ }^{64}$.

Keefer et al. ${ }^{13}$ abordaram o revestimento de microeletrodos com NTCs e demonstraram redução da impedância e aumento da condutividade elétrica, com manutenção da inércia química e da biocompatibilidade. Os eletrodos modificados tiveram sua capacidade de ativar neurônios aumentada em culturas e em experimentos com ratos e macacos, sugerindo que o revestimento com NTCs é permissivo para o crescimento neuronal e manutenção funcional por pelo menos três meses, são estáveis sob condições fisiológicas e aumentam a sensibilidade da estimulação neuronal.

A capacidade de potencializar a excitabilidade neuronal confere aos NTCs grande aplicabilidade para regeneração de neurônios em lesões cerebrais ${ }^{92}$. Além das promissoras respostas sinápticas obtidas em cultura, eles apresentam importante diferencial que favorece o interfaciamento biológico: seu tamanho. A escala não nanométrica é limitada, pois gera um sinal bioativo homogêneo em toda a extensão neuronal. As nanoestruturas possibilitam estímulos com resoluções maiores, em níveis subcelulares, o que garante a especificidade do sinal93.

$\mathrm{O}$ recente progresso de estudos envolvendo a aplicação da nanotecnologia em interfaces bioelétricas com células do SNC tem significativas implicações para a terapêutica de DNs. Interfaces neurais em dimensões nanométricas permitem aumentar a especificidade do estímulo e reduzir os efeitos colaterais, tais como resposta fibrótica local, associados aos dispositivos micrométricos atualmente empregados ${ }^{94}$. No entanto, o mecanismo envolvido na interação nanotubo-neurônio permanece incógnito, sendo necessários mais estudos.

\section{Discussão}

Os NTCs têm grande potencial para aplicações na biotecnologia e na neuromedicina, podendo atuar no carreamento de fármacos, genes ou biomoléculas, como suporte para o crescimento neuronal e como interfaces elétricas para estímulo da atividade sináptica. Tais aplicações conferem aos NTCs a chave para o tratamento de DNs. No entanto, vários pontos relativos à estrutura, biocompatibilidade e mecanismos de interação nanotubo-neurônio permanecem sem esclarecimento, o que impede que tais materiais sejam mais bem explorados científica e comercialmente.

Portanto, faz-se imprescindível ampliar o conhecimento acerca dos processos de síntese e purificação dos NTCs, a fim de garantir produtos finais livres de resíduos catalíticos e defeitos estruturais, bem como adequar a funcionalização às necessidades celulares, possibilitando melhores condições de crescimento, adesão e interação das culturas neuronais com o substrato. A padronização dos ensaios de toxicidade dos nanomateriais também é fundamental para que conclusões definitivas a respeito da sua biocompatibilidade sejam alcançadas. Os mecanismos de interação nanotubo-neurônio também precisam ser elucidados, sendo essenciais avaliações histológicas da região em contato com o implante nanométrico para caracterizar a biocompatibilidade em longo prazo.

Os avanços nessas questões dependem de abordagens científicas multidisciplinares, combinando o conhecimento clínico com as técnicas de síntese e funcionalização dos NTCs. Se essas áreas trabalharem de forma integrada para o desenvolvimento da nanoneuromedicina, dispositivos implantáveis chegarão à clínica brevemente, revolucionando as condutas terapêuticas atuais e conferindo novas perspectivas aos pacientes com DNs.

\section{Conclusão}

Os estudos envolvendo a aplicação dos NTCs nas neurociências são promissores. Apesar de ser um novo material, os ensaios apontam uma revolução nas áreas diagnósticas e terapêuticas das DNs. Pela necessidade de maior compreensão dos mecanismos de interação dessas nanoestruturas com sistemas biológicos, bem como sua biocompatibilidade, mais estudos são necessários para ampliar a sua utilização no campo das neurociências.

\section{Agradecimentos}

FAPEMIG (Edital 17/2010 Pronex CBB - APQ-04334-10), CAPES (Edital 04/CII-2008 - Rede NANOBIOTEC-Brasil), Rede AGRONANO e FAPESP. 


\section{Referências}

1. ISO - International Organization for Standardization. ISO/TC 229: Nanotechnologies. ISO/TS 80004-1. 2010. Disponível em: https://cdb. iso.org/cdb/termentry!display.action?entry $=497541$ \&language $=1$.

2. Lewinski N, Colvin V, Drezek R. Cytotoxicity of nanoparticles. Small. 2008;4(1):26-49.

3. Chen X, Schluesener HJ. Nanosilver: a nanoproduct in medical application. Toxicol. Lett. 2008;176(1):1-12.

4. Emerich DF. Nanomedicine - prospective therapeutic and diagnostic applications. Expert Opin Biol Ther. 2005;5(1):1-5.

5. Chan WC. Bionanotechnology progress and advances. Biol Blood Marrow Transplant. 2006;12(1):87-91.

6. Shaffer C. Nanomedicine transforms drug delivery. Drug Discov Today. 2005;10(23-24):1581-2.

7. Sahoo SK, Parveen S, Panda JJ. The present and future of nanotechnology in human health care. Nanomedicine. 2007;3(1):20-31.

8. Moore MN. Do nanoparticles present ecotoxicological risks for the health of the aquatic environment? Environ Int. 2006;32(8):967-76.

9. Paschoalino MP, Marcone GPS, Jardim WF. Os nanomateriais e a questão ambiental. Quím Nova. 2010;33(2):421-30.

10. Zarbin AJG. Química de (nano)materiais. Quím Nova. 2007;30(6):1469-79.

11. Malarkey EB, Parpura V. Carbon nanotubes in neuroscience. Acta Neurochir Suppl. 2010;106:337-41.

12. Hochberg LR, Serruya MD, Friehs GM, Mukand JA, Saleh M, Caplan $\mathrm{AH}$, et al. Neuronal ensemble control of prosthetic devices by a human with tetraplegia. Nature. 2006;442(7099):164-71.

13. Keefer EW, Botterman BR, Romero MI, Rossi AF, Gross GW. Carbon nanotube coating improves neuronal recordings. Nat Nanotechnol. 2008;3(7):434-9.

14. Malarkey EB, Parpura V. Applications of carbon nanotubes in neurobiology. Neurodegener Dis. 2007;4(4):292-9.

15. Wei W, Sethuraman A, Jin C, Monteiro-Riviere NA, Narayan RJ. Biological properties of carbon nanotubes. J Nanosci Nanotechnol. 2007;7(4):1284-97.

16. Benabid AL, Wallace B, Mitrofanis J, Xia C, Piallat B, Fraix V, et al. Therapeutic electrical stimulation of the central nervous system. C R Biol. 2005;328(2):177-86.

17. Lovat V, Pantarotto D, Lagostena L, Cacciari B, Grandolfo M, Righi M, et al. Carbon nanotube substrates boost neuronal electrical signaling. Nano Lett. 2005;5(6):1107-10.

18. Lebedev MA, Nicolelis MA. Brain-machine interfaces: past, present future. Trends Neurosci. 2006;29(9):536-46.

19. Mussa-ivaldi FA, Miller LE. Brain-machine interfaces: computational demands clinical needs meet basic neuroscience. Trends Neurosci. 2003;26(6):329-34.

20. Vergoni AV, Tosi G, Tacchi R, Vandelli MA, Bertolini A, Costantino, L. Nanoparticles as drug delivery agents specific for CNS: in vivo biodistribution. Nanomedicine. 2009;5(4):369-77.

21. Fagan SB, Silva AJR, Mota R, Baierle RJ, Fazzio A. Functionalization of carbon nanotubes through the chemical binding of atoms and molecules. Phys Rev B. 2003;67(3):033405-8.

22. Bardi G, Tognini P, Ciofani G, Raffa V, Costa M, Pizzorusso T. Pluronic-coated carbon nanotubes do not induce degeneration of cortical neurons in vivo and in vitro. Nanomedicine. 2009;5(1):96-104.

23. Baughman RH, Zakhidov AA, De Heer WA. Carbon nanotubes - the route toward applications. Science. 2002;297(5582):787-92.

24. Lima MD, Bonadiman R, De Andrade MJ, Toniolo J, Bergmann CP. Synthesis of multi-walled carbon nanotubes by catalytic chemical vapor deposition using $\mathrm{Cr} 2 \mathrm{xFexO} 3$ as catalyst. Diam Relat Mater. 2006:15(10):1708-13.

25. Belyanskaya L, Weigel S, Hirsch C, Tobler U, Krug HF, Wick P. Effects of carbon nanotubes on primary neurons and glial cells. Neurotoxicology. 2009;30(4):702-11.

26. Liu JM, Rao Y, Chen L, Wang XX, Lin LP, Lin CQ, et al. A new phosphorimetry for the determination of trace alkaline phosphatase using multi-wall carbon nanotubes and its diagnosis of human diseases. J Fluoresc. 2010;20(4):933-41.

27. Wan AC, Ying JY. Nanomaterials for in situ cell delivery and tissue regeneration. Adv Drug Deliv Rev. 2010;62(7-8):731-40.
28. Bianco A, Kostarelos K, Prato M. Applications of carbon nanotubes in drug delivery. Curr Opin Chem Biol. 2005;9(6):674-9.

29. Kam NWS, O'Connell M, Wisdom JA, Dai H. Carbon nanotubes as multifunctional biological transporters and near-infrared agents for selective cancer cell destruction. Proc Natl Acad Sci. 2005;102(33):11600-5.

30. Im JS, Bai BC, Lee Y. The effect of carbon nanotubes on drug delivery in an electro-sensitive transdermal drug delivery system. Biomaterials. 2010;31(6):1414-9.

31. Cheung W, Pontoriero F, Taratula O, Chen AM, He H. DNA and carbon nanotubes as medicine. Adv Drug Deliv Rev. 2010;62(6):633-49.

32. Lu Q, Moore JM, Huang G, Mount AS, Rao AM, Larcom LL, et al. RNA polymer translocation with single-walled carbon nanotubes. Nano Lett. 2004;4(12):2473-7.

33. Sinnott SB. Chemical functionalization of carbon nanotubos. J Nanosci Nanotechnol. 2002;2(2):113-23.

34. Pantarotto D, Singh R, Mccarthy D, Erhardt M, Briand JP, Prato M, et al. Functionalized carbon nanotubes for plasmid DNA gene delivery. Angew Chem Int Ed. 2004;43(39):5242-6.

35. Patlolla A, Patlolla B, Tchounwou P. Evaluation of cell viability, DNA damage, and cell death in normal human dermal fibroblast cells induced by functionalized multiwalled carbon nanotube. Mol Cell Biochem. 2009;338(1-2):225-32.

36. Sayes CM, Liang F, Hudson JL, Mendez J, Guo W, Beach JM, et al. Functionalization density dependence of single-walled carbon nanotubes cytotoxicity in vitro. Toxicol Lett. 2006;161(2):135-42.

37. Bottini M, Bruckner S, Nika K, Bottini N, Bellucci S, Magrini A, et al. Multi-walled carbon nanotubes induce T lymphocyte apoptosis. Toxicol Lett. 2006;160(2):121-6.

38. Hussain MA, Kabir MA, Sood AK. On the cytotoxicity of carbon nanotubes. Curr Sci. 2009;96(5):664-73.

39. Manna SK, Sarkar S, Barr J, Wise K, Barrera EV, Jejelowo O, et al. Singlewalled carbon nanotube induces oxidative stress and activates nuclear transcription factor- $\mathrm{\kappa B}$ in human keratinocytes. Nano Lett. 2005;5(9):1676-84.

40. Poland CA, Duffin R, Kinloch I, Maynard A, Wallace WAH, Seaton A, et al. Carbon nanotubes introduced into the abdominal cavity of mice show asbestos-like pathogenicity in a pilot study. Nat Nanotechnol. 2008;3(7):423-8.

41. Reddy AR, Reddy YN, Krishna DR, Himabindu V. Multi wall carbon nanotubes induce oxidative stress and cytotoxicity in human embryonic kidney (HEK293) cells. Toxicology. 2010; 272(1-3):11-6.

42. Belyanskaya L, Manser P, Spohn P, Bruinink A, Wic P. The reliability and limits of the MTT reduction assay for carbon nanotubes-cell interaction. Carbon. 2007;45(13):2643-8.

43. De Nicola M, Nuccitelli S, Gattia DM, Traversa E, Magrini A, Bergamaschi A, et al. Effects of carbon nanotubes on human monocytes. Ann N Y Acad Sci. 2009;1171(1):600-5.

44. Lam CW, James JT, McCluskey R, Hunter RL. Pulmonary toxicity of carbon nanotubes in mice 7 and 90 days after intratracheal instillation. Toxicol Sci. 2003;72(1):126-34.

45. Shvedova AA, Kisin ER, Mercer R, Murray AR, Johnson VJ, Potapovich $\mathrm{AI}$, et al. Unusual inflammatory and fibrogenic pulmonary responses to single-walled carbon nanotubes in mice. Am J Physiol Lung Cell Mol Physiol. 2005;289(5):698-708.

46. Li Z, Hulderman T, Salmen R, Chapman R, Leonard SS, Young SH, et al. Cardiovascular effects of pulmonary exposure to single-wall carbon nanotubes. Environ Health Perspect. 2007;115(3):377-82.

47. Shvedova AA, Castranova V, Kisin ER, Schwegler-Berry D, Murray AR, Gandelsman VZ, et al. Exposure to carbon nanotube material: assessment of nanotube cytotoxicity using human keratinocyte cells. J Toxicol Environ Health A. 2003;66(20):1909-26.

48. Yehia HN, Draper RK, Mikoryak C, Walker EK, Baj AP, Musselman $\mathrm{IH}$, et al. Single-walled carbon nanotube interactions with HeLa cells. J Nanobiotechnology. 2007;5(8):1-17.

49. Huczko A, Lange H. Fullerene. Sci Technol. 2001;9:247-50.

50. Zhang LW, Zeng L, Barron AR, Monteiro-Riviere NA. Biological interactions of functionalized single-wall carbon nanotubes in human epidermal keratinocytes. Int J Toxicol. 2007;26(2):103-13.

51. Wang Y, Iqbal Z, Mitra S. Rapidly functionalized, water dispersed carbon nanotubes at high concentration. J Am Chem Soc. 2006;128(1):95-9. 
52. Huang W, Fernando S, Lin Y, Zhou B, Allard LF, Sun Ya-Ping. Preferential solubilization of smaller single-walled carbon nanotubes in sequential functionalization reactions. Langmuir. 2003;19(17):7084-8.

53. Kagan VE, Bayir HB, Shvedova AA. Nanomedicine and nanotoxicology: two sides of the same coin. Nanomedicine. 2005;1(4):313-6.

54. Magrez A, Kasas S, Salicio V, Pasquier N, Seo JW, Celio M, et al. Cellular toxicity of carbon-based nanomaterials. Nano Lett. 2006;6(6):1121-5.

55. Wick P, Manser P, Limbach LK, Dettlaff-Weglikowska U, Krumeich F, Roth S, et al. The degree and kind of agglomeration affect carbon nanotube cytotoxicity. Toxicol Lett. 2007;168(2):121-31.

56. Pulskamp K, Diabaté S, Krug HF. Carbon nanotubes show no sign of acute toxicity but induce intracellular reactive oxygen species in dependence on contaminants. Toxicol Lett. 2007;168(1):58-74.

57. Fenoglio I, Tomatis M, Lison D, Muller J, Fonseca A, Nagy JB, et al. Reactivity of carbon nanotubes: free radical generation or scavenging activity. Free Radic Biol Med. 2006;40(7):1227-33.

58. Tosi G, Costantino L, Rivasi F, Ruozi B, Leo E, Vergoni AV, et al. Targeting the central nervous system: in vivo experiments with peptide derivatized nanoparticles loaded with Loperamide and Rhodamine. J Control Release. 2007;122(1):1-9.

59. Oberdörster G, Sharp Z, Atudorei V, Elder A, Gelein R, Kreyling W, et al. Translocation of inhaled ultrafine particles to the brain. Inhal Toxicol. 2004;16(6-7):437-45.

60. Peters A, Veronesi B, Calderón-Garcidueñas L, Gehr P, Chen LC, Geiser $\mathrm{M}$, et al. Translocation and potential neurological effects of fine and ultrafine particles a critical update. Part Fibre Toxicol. 2006;3(13):1-13.

61. Oberdörster G, Maynard A, Donaldson K, Castranova V, Fitzpatrick J, Ausman K, et al. Principles for characterizing the potential human health effects from exposure to nanomaterials: elements of a screening strategy. Part Fibre Toxicol. 2005;2(8):1-35.

62. Hu H, Ni Y, Montana V, Haddon RC, Parpura V. Chemically functionalized carbon nanotubes as substrates for neuronal growth. Nano Lett. 2004;4(3):507-11.

63. Mattson MP, Haddon RC, Rao AM. Molecular functionalization of carbon nanotubes and use as substrates for neuronal growth. J Mol Neurosci. 2000;14(3):175-82.

64. Jakubek LM, Marangoudakis S, Raingo J, Liu X, Lipscombe D, Hurt RH. The inhibition of neuronal calcium ion channels by trace levels of yttrium released from carbon nanotubes. Biomaterials. 2009;30(31):6351-7.

65. Mazzatenta A, Giugliano M, Campidelli S, Gambazzi L, Businaro L, Markram $\mathrm{H}$, et al. Interfacing neurons with carbon nanotubes: electrical signal transfer and synaptic stimulation in cultured brain circuits. J Neurosci. 2007;279(26):6931-6.

66. Modi G, Pillay V, Choonara YE. Advances in the treatment of neurodegenerative disorders employing nanotechnology. Ann N Y Acad Sci. 2010;1184:154-72.

67. Flachenecker P. Epidemiology of neuroimmunological diseases. J Neurol. 2006;253: 2-8.

68. De Boer AG, Gaillard PJ. Drug targeting to the brain. Annu Rev Pharmacol Toxicol. 2007;47:323-55.

69. Forlenza OV. Tratamento farmacológico da doença de Alzheimer. Rev Psiq Clín. 2005;32(3):137-48.

70. Nitrini R. Há sentido em utilizar o tratamento atualmente disponível para a doença de Alzheimer? Rev Psiq Clín. 2006;33(4):214-7.

71. Hyman BT. The neuropathological diagnosis of Alzheimer's disease: clinical-pathological studies. Neurobiol Aging. 1997;18(4):27-32.

72. Seil JT, Webster TJ. Electrically active nanomaterials as improved neural tissue regeneration scaffolds. Wiley Interdiscip Rev Nanomed Nanobiotechnol. 2010;2(6):635-47.
73. Merkus P, Guchelaar HJ, Bosch DA, Merkus FW. Direct access of drugs to the human brain after intranasal drug administration? Neurology. 2003;60(10):1669-71.

74. Kanwar JR, Mahidhara G, Kanwar RK. Recent advances in nanoneurology for drug delivery to the brain. Curr Nanosci. 2009;5(4):441-8.

75. Abidian MR, Kim DH, Martin DC. Conducting-polymer nanotubes for controlled drug release. Adv Mater. 2006;18:405-9.

76. Modi G, Pillay V, Choonara YE, Ndesendo VMK, Du Toit LC, Naidoo D. Nanotechnological applications for the treatment of neurodegenerative disorders. Prog Neurobiol. 2009;88(4):272-85.

77. Behan N, Birkinshaw C, Clarke N. Poly n-butyl cyanoacrylate nanoparticles: a mechanistic study of polymerization and particle formation. Biomaterials. 2001;22(11):1335-44.

78. Muller RH, Keck CM. Drug delivery to the brain-realization by novel drug carriers. J Nanosci Nanotechnol. 2004;4(5):471-83.

79. Oishi M, Hayashi H, Iijima M, Nagasaki Y. Endosomal release and intracellular delivery of anticancer drugs using $\mathrm{pH}$-sensitive pegylated nanogels. J Mater Chem. 2007;17:3720-5.

80. Gosk S, Vermehren C, Storm G, Moos, T. Targeting anti-transferrin receptor antibody (OX26) and OX26-conjugated liposomes to brain capillary endothelial cells using in situ perfusion. J Cereb Blood Flow Metab. 2004;24(11):1193-204.

81. Tran PA, Zhang L, Webster TJ. Carbon nanofibers and carbon nanotubes in regenerative medicine. Adv Drug Deliv Rev. 2009;61(12):1097-114.

82. Shi Kam NW, Jessop TC, Wender PA, Dai H. Nanotube molecular transporters: internalization of carbon nanotube protein conjugates into mammalian cells. J Am Chem Soc. 2004;126(22):6850-1.

83. Subramanian A, Krishnan UM, Sethuraman S. Development of biomaterial scaffold for nerve tissue engineering: biomaterial mediated neural regeneration. J Biomed Sci. 2009;16(1):108.

84. Jan E, Kotov NA. Successful differentiation of mouse neural stem cells on layer-by-layer assembled single-walled carbon nanotube composite. Nano Lett. 2007;7(5):1123-8.

85. Liopo AV, Stewart MP, Hudson J, Tour JM, Pappas TC. Biocompatibility of native functionalized single-walled carbon nanotubes for neuronal interface. J Nanosci Nanotechnol. 2006;6(5):1365-74.

86. Matsumoto K, Sato C, Naka Y, Kitazawa A, Whitby RL, Shimizu N. Neurite outgrowths of neurons with neurotrophin-coated carbon nanotubes. J Biosci Bioeng. 2007;103(3):216-20.

87. Powell SK, Rao J, Roque E, Nomizu M, Kuratomi Y, Yamada Y, et al. Neural cell response to multiple novel sites on laminin-1. J Neurosci Res. 2000;61(3):302-12.

88. Du Toit DF, Kleintjes WG, Mazyala EJ, Bhatia DN, De Beer JF, Page BJ. Shoulder surgeon and autologous cellular regeneration - From bench to bed: Part one: the link between the human fibroblast, connective tissue disorders and shoulder. Int J Shoulder Surg. 2007;1(3):87-95.

89. Mayberg HS, Lozano AM, Voon V, McNeely HE, Seminowicz D, Hamani C, et al. Deep brain stimulation for treatment-resistant depression. Neuron. 2005;(45):651-60.

90. Pancrazio JJ. Neural interfaces at the nanoscale. Nanomedicine. 2008;3(6):823-30.

91. Cellot G, Cilia E, Cipollone S, Rancic V, Sucapane A, Giordani S, et al. Carbon nanotubes might improve neuronal performance by favouring electrical shortcuts. Nat Nanotech. 2009;4(2):126-33.

92. Lee W, Parpura V. Wiring neurons with carbon nanotubes. Front Neuroengering. 2009;2(8):1-3.

93. Silva GA. Neuroscience nanotechnology: progress, opportunities and challenges. Nat Rev. 2006;7(1):65-74.

94. Mckenzie JL, Waid MC, Shi R, Webster TJ. Decreased functions of astrocytes on carbon nanofiber materials. Biomaterials. 2004;25(7-8):1309-17. 